\title{
The notable configuration of inscribed equilateral triangles in a triangle
}

\author{
Blas Herrera Gómez \\ Blas Herrera Gómez obtained his Ph.D. in mathematics at the University Autònoma \\ of Barcelona in 1994. Presently, he is a professor at the University Rovira i Virgili of \\ Tarragona. His main fields of interest are: the geometry of foliations, the dynamics \\ of galaxies, classical geometry, and the mechanics of fluids and turbulence.
}

\section{Introduction}

Many notable configurations for a given triangle have been described by different geometers in the past. Among the most famous classic configurations are the Euler and Simson lines, the Gergonne, Lemoine, Brocard and Nagel concurrences, the Feuerbach, Brocard, Lemoine, Tuker and Taylor circumferences, etc. More modern configurations can be found in the Soddy circles [9], the Euler-Gergonne-Soddy triangle [8], the Torricelli configuration [10], etc. In fact, one can see in [3] four hundred configurations (triangle centers), in [4] even eight hundred configurations are picked up. Recently, new configurations continue to appear, for example Morley triangles related to the Feuerbach circumferences [5], associated rectangular hyperbolas [1], triangle centers associated with a rhombus [6], Euler lines concurrent on the nine-point circle [7], etc.

Among modern configurations we focus our attention on the Lucas circles. Edouard Lucas studied the configuration given by three inscribed squares in a triangle with a side parallel to each one of the sides of the triangle. With them appear three circles. Recently [2], Yiu and Hatzipolakis have been continuing the study of this configuration and, in particular, have shown recently that the Lucas circles are tangent to each other.

Bekanntlich gibt es eine Vielzahl bereits untersuchter Konfigurationen zu einem gegebenen ebenen Dreieck. Der Autor des nachfolgenden Beitrags knüpft an eine von E. Lucas studierte Konfiguration an, die ein Dreieck mit drei einbeschriebenen Quadraten zum Ausgangspunkt hat und zu den sogenannten Lucas-Kreisen führt. Hier wird nun ein Dreieck mit drei einbeschriebenen gleichseitigen Dreiecken, von denen jeweils genau eine Seite zu einer der Seiten des Ausgangsdreiecks parallel ist, untersucht. Bei dieser Konfiguration zeigt sich unter anderem, dass jeweils vier der insgesamt neun Eckpunkte der einbeschriebenen Dreiecke auf drei Kreisen liegen, die sich in einem Punkt schneiden. 
In this paper we take on Lucas' idea and consider not the three inscribed squares, but the three inscribed equilateral triangles with one side parallel to each one of the sides of the triangle (see Fig. 1).

The notable configuration which appears is outlined below and will be summarized in the theorem of the following section.

We will show that the centers of these equilateral triangles are aligned (see Fig. 2). We will see also that the mid-points of the sides of the equilateral triangles are aligned three by three (see Fig. 2). We will find that the simple ratios of these mid-points and the simple ratios of the vertices are equal to the simple ratio of the centers. But the most remarkable feature will be the appearance of three circumferences which link the vertices four by four, and these three circles are concurrent in a point (see Figs. 3, 4).

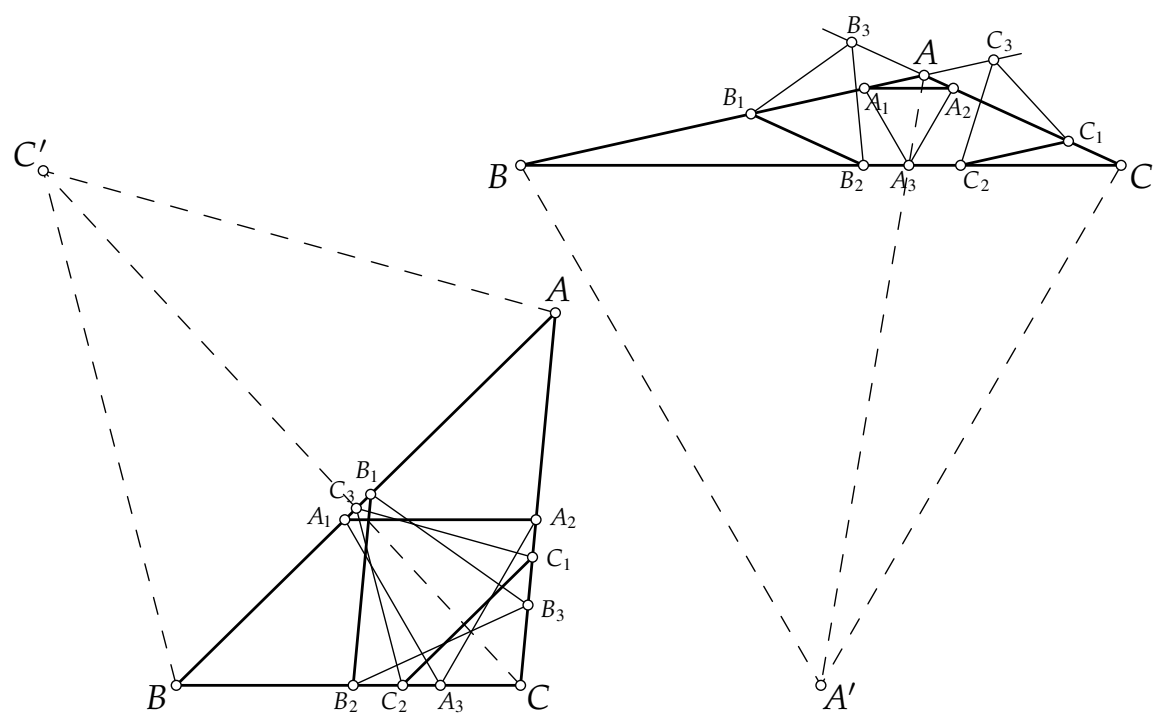

Fig. 1 Construction of the three inscribed equilateral triangles.

\section{Configuration}

First of all, in order to establish our notations we recall that the "simple ratio" $(A, B, C)$ of three aligned points is $(A, B, C)= \pm \frac{A C}{B C}$ with a positive sign if $C$ is not between $A$ and $B$, and a negative sign otherwise.

We recall that in order to construct an inscribed equilateral triangle in $\triangle A B C$ with a side parallel to side $A B$, we consider the exterior equilateral triangle with side $A B, \triangle A B C^{\prime}$. Let $C_{3}$ be the intersection of the line $C C^{\prime}$ with the line $A B$. The lines through the point $C_{3}$ parallel to $A C^{\prime}$ and $B C^{\prime}$ intersect with the lines $A C$ and $B C$ at the points $C_{1}$ and $C_{2}$ respectively. The triangle $\triangle C_{1} C_{2} C_{3}$ is the inscribed equilateral triangle (see Fig. 1).

The other two triangles $\triangle A_{1} A_{2} A_{3}$ and $\triangle B_{1} B_{2} B_{3}$, with sides parallel to $B C$ and $A C$ respectively, can be constructed in a similar way.

The description of the configuration can be summarized in the following theorem. 


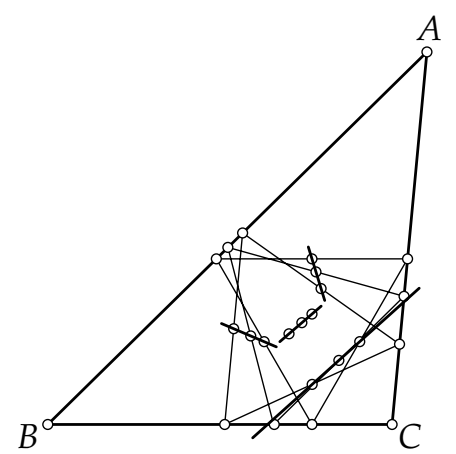

Fig. 2 The centers $O_{A}, O_{B}, O_{C}$ are aligned. The lines $A A_{3}, B B_{3}, C C_{3}$ are concurrent (outward Fermat point $F=X_{13}$ ). The mid-points $A_{12}, B_{13}, C_{13}$ are aligned; the mid-points $A_{13}, B_{12}, C_{23}$ are aligned; the midpoints $A_{23}, C_{12}, B_{23}$ are aligned. All the simple ratios coincide with the simple ratio $\left(O_{A}, O_{B}, O_{C}\right)$.

Theorem 1 Let $\triangle A B C$ be a triangle. Let $\triangle A_{1} A_{2} A_{3}, \triangle B_{1} B_{2} B_{3}, \triangle C_{1} C_{2} C_{3}$ be the three inscribed equilateral triangles with $A_{1} A_{2}$ parallel to $B C, B_{1} B_{2}$ parallel to $A C, C_{1} C_{2}$ parallel to $A B$ and $A_{1}, B_{1}, C_{3}$ on the line $A B$.

Let $O_{A}, O_{B}, O_{C}$ be the respective centers of the three equilateral triangles, and let $A_{i j}$, $B_{i j}, C_{i j}$ be the respective mid-points of the sides $A_{i} A_{j}, B_{i} B_{j}, C_{i} C_{j}$.

Then we have:

1. The center points $O_{A}, O_{B}, O_{C}$ are aligned.

2. The lines $A A_{3}, B B_{3}, C C_{3}$ are concurrent (in the outward Fermat point).

3. The mid-points $A_{12}, B_{13}, C_{13}$ are aligned, the mid-points $A_{13}, B_{12}, C_{23}$ are aligned and the mid-points $A_{23}, C_{12}, B_{23}$ are also aligned.

4. The following simple ratios are equal:

$$
\begin{aligned}
\left(O_{A}, O_{B}, O_{C}\right) & =\left(A_{2}, B_{3}, C_{1}\right)=\left(A_{1}, B_{1}, C_{3}\right)=\left(A_{3}, B_{2}, C_{2}\right) \\
& =\left(A_{12}, B_{13}, C_{13}\right)=\left(A_{13}, B_{12}, C_{23}\right)=\left(A_{23}, B_{23}, C_{12}\right) .
\end{aligned}
$$

5. The points $A_{3}, C_{2}, A_{1}, C_{3}$ are concyclic, the points $A_{3}, B_{2}, A_{2}, B_{3}$ are concyclic and the points $C_{3}, B_{1}, C_{1}, B_{3}$ are also concyclic.

6. The above circumferences $A_{3} C_{2} A_{1} C_{3}, A_{3} B_{2} A_{2} B_{3}, C_{3} B_{1} C_{1} B_{3}$ are concurrent in a point $\eta$.

Proof. Take a system of Cartesian coordinates in such a way that

$$
A=(a, b) \text { with } 0 \leq a, b \leq 1, B=(0,0), C=(1,0) .
$$

A straightforward computation proves the parts 1 and 3 of the theorem (see Fig. 2).

Part 2 of the theorem is well-known. The concurrence of the lines $A A_{3}, B B_{3}, C C_{3}$ is the outward Fermat point $F\left(X_{13}\right.$ in [4]). 


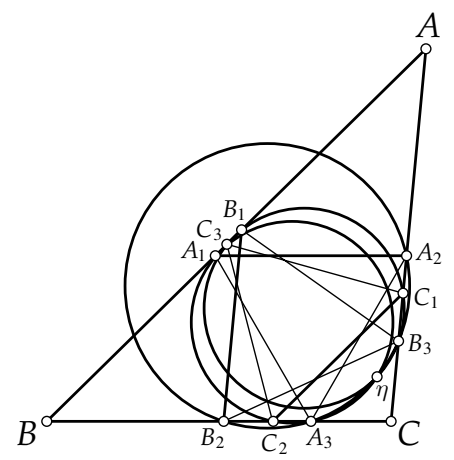

Fig. 3 The points $A_{3}, C_{2}, A_{1}$ and $C_{3}$ are concyclic, the points $A_{3}, B_{2}, A_{2}$ and $B_{3}$ are concyclic and the points $C_{3}, B_{1}, C_{1}$ and $B_{3}$ are concyclic. Also, the three circumferences are concurrent at the point $\eta$.

Part 4 can be found with a straightforward computation. The value of the simple ratio obtaining in every case is the same expression:

$$
\left(O_{A}, O_{B}, O_{C}\right)=\frac{-1}{\sqrt{3}} \frac{(3 a-\sqrt{3} b)\left(3 a^{2}-6 a+3 b^{2}+2 \sqrt{3} b+3\right)}{(2 b+\sqrt{3})\left(3 a^{2}-3 a+3 b^{2}-\sqrt{3} b\right)} .
$$

Part 5 (see Figs. 3, 4) can be found with a long computation and we have that the points $A_{3}, C_{2}, A_{1}, C_{3}$ belong to the circumference of center $O_{A C}$ given by

$$
\begin{aligned}
& O_{A C x}=\frac{1}{2} \sqrt{3} \frac{3 a^{3}+3 \sqrt{3} a^{2} b+3 a b^{2}+3 a^{2}+2 \sqrt{3} a b+3 \sqrt{3} b^{3}+5 b^{2}}{\left(3 a^{2}+3 b^{2}+2 \sqrt{3} b\right)(2 b+\sqrt{3})} \\
& O_{A C y}=-\frac{1}{2} \frac{3 a^{3}-5 \sqrt{3} a^{2} b+3 a b^{2}-3 a^{2}+2 \sqrt{3} a b-5 \sqrt{3} b^{3}-9 b^{2}}{\left(3 a^{2}+3 b^{2}+2 \sqrt{3} b\right)(2 b+\sqrt{3})}
\end{aligned}
$$

and radius $r_{A C}$ :

$$
\sqrt{\frac{\left(a^{2}+b^{2}\right)\left(3 a^{4}-4 \sqrt{3} a^{3} b+10 a^{2} b^{2}-4 \sqrt{3} a b^{3}-6 a^{3}+8 \sqrt{3} a^{2} b-14 a b^{2}+3 a^{2}-4 \sqrt{3} a b+7 b^{4}+8 \sqrt{3} b^{3}+7 b^{2}\right)}{\left(3 a^{2}+3 b^{2}+2 \sqrt{3} b\right)^{2}(2 b+\sqrt{3})^{2}}} .
$$

Analogously, the points $A_{3}, B_{2}, A_{2}, B_{3}$ belong to the circumference of center $O_{A B}$ given by:

$$
\begin{aligned}
& O_{A B x}=\frac{1}{2} \sqrt{3} \frac{3 a^{3}+\sqrt{3} a^{2} b+3 a b^{2}-6 a^{2}+3 a+\sqrt{3} b^{3}+6 b^{2}+3 \sqrt{3} b}{\left(3 a^{2}-6 a+3 b^{2}+2 \sqrt{3} b+3\right)(2 b+\sqrt{3})} \\
& O_{A B y}=\frac{1}{2} \frac{3 a^{3}+5 \sqrt{3} a^{2} b+3 a b^{2}-6 a^{2}-8 \sqrt{3} a b+3 a+5 \sqrt{3} b^{3}+6 b^{2}+3 \sqrt{3} b}{\left(3 a^{2}-6 a+3 b^{2}+2 \sqrt{3} b+3\right)(2 b+\sqrt{3})}
\end{aligned}
$$




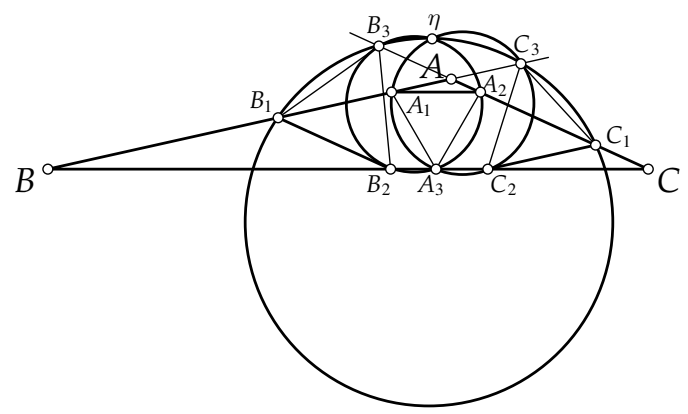

Fig. 4 Concurrence of the three circumferences.

and radius $r_{A B}$ :

$$
\sqrt{3 \frac{\left(a^{2}-2 a+b^{2}+1\right)\left(3 a^{4}+4 \sqrt{3} a^{3} b+10 a^{2} b^{2}+4 \sqrt{3} a b^{3}-6 a^{3}-4 \sqrt{3} a^{2} b-6 a b^{2}+3 a^{2}+7 b^{4}+4 \sqrt{3} b^{3}+3 b^{2}\right)}{\left(3 a^{2}-6 a+3 b^{2}+2 \sqrt{3} b+3\right)^{2}(2 b+\sqrt{3})^{2}}} .
$$

Analogously the points $B_{1}, C_{3}, B_{3}, C_{1}$ belong to the circumference of center $O_{B C}$ given by:

$$
\begin{aligned}
& O_{B C x}=\frac{3}{2} \frac{3 a^{4}+4 \sqrt{3} a^{3} b+6 a^{2} b^{2}+4 \sqrt{3} a b^{3}-6 a^{3}-2 \sqrt{3} a^{2} b-2 a b^{2}+3 a^{2}+2 \sqrt{3} a b+3 b^{4}+2 \sqrt{3} b^{3}+5 b^{2}}{\left(3 a^{2}+2 \sqrt{3} b+3 b^{2}\right)\left(3 a^{2}-6 a+3+2 \sqrt{3} b+3 b^{2}\right)}, \\
& O_{B C y}=-\frac{1}{2} \frac{\sqrt{3}\left(3 a^{2}-3 a+3 b^{2}+\sqrt{3} b\right)(a-\sqrt{3} b-1)(\sqrt{3} b+a)}{\left(3 a^{2}+3 b^{2}+2 \sqrt{3} b\right)\left(3 a^{2}-6 a+3 b^{2}+2 \sqrt{3} b+3\right)}
\end{aligned}
$$

and radius $r_{B C}$ :

$$
\sqrt{9 \frac{\left(a^{2}+b^{2}\right)\left(a^{2}-2 a+b^{2}+1\right)\left(3 a^{4}+6 a^{2} b^{2}-6 a^{3}-4 \sqrt{3} a^{2} b-6 a b^{2}+3 a^{2}+4 \sqrt{3} a b+3 b^{4}+4 \sqrt{3} b^{3}+7 b^{2}\right)}{\left(3 a^{2}+3 b^{2}+2 \sqrt{3} b\right)^{2}\left(3 a^{2}-6 a+3 b^{2}+2 \sqrt{3} b+3\right)^{2}}} .
$$

Finally, part 6 corresponds to the above circumferences

$$
A_{3} C_{2} A_{1} C_{3}, \quad A_{3} B_{2} A_{2} B_{3}, \quad C_{3} B_{1} C_{1} B_{3}
$$

are concurrent in a point $\eta$ (see Figs. 3, 4). To prove this statement let $\eta$ be the image of point $A_{3}$ under the axial symmetry with respect to the line $O_{A B} O_{A C}$. We obtain

$$
\eta=\left(\frac{1}{2} \frac{\varsigma}{\tau},-\frac{1}{6} \sqrt{3} \frac{v}{\phi}\right)
$$


with

$$
\begin{aligned}
& \varsigma=3 a^{6}+8 \sqrt{3} a^{5} b+9 a^{4} b^{2}+16 \sqrt{3} a^{3} b^{3}+9 a^{2} b^{4}+8 \sqrt{3} a b^{5}-3 a^{5} \\
& -11 \sqrt{3} a^{4} b-6 a^{3} b^{2}-14 \sqrt{3} a^{2} b^{3}-3 a b^{4}-3 a^{4}-2 \sqrt{3} a^{3} b \\
& -10 \sqrt{3} a b^{3}+3 a^{3}+5 \sqrt{3} a^{2} b+3 a b^{2}+3 b^{6}-3 \sqrt{3} b^{5}-5 b^{4}+5 \sqrt{3} b^{3}, \\
& \tau=3 a^{6}+9 a^{4} b^{2}+9 a^{2} b^{4}+9 \sqrt{3} a^{4} b-9 a^{5}-18 a^{3} b^{2}+10 \sqrt{3} a^{2} b^{3} \\
& -9 a b^{4}+12 a^{4}-18 \sqrt{3} a^{3} b+18 a^{2} b^{2}-10 \sqrt{3} a b^{3}-9 a^{3}+9 \sqrt{3} a^{2} b \\
& +3 a^{2}-9 a b^{2}+3 b^{6}+\sqrt{3} b^{5}-2 b^{4}+\sqrt{3} b^{3}+3 b^{2}, \\
& v=\left(3 a^{2}-3 a+3 b^{2}-\sqrt{3} b\right)\left(3 a^{4}-2 a^{2} b^{2}-6 a^{3}+2 a b^{2}+3 a^{2}-5 b^{4}+3 b^{2}\right) \text {, } \\
& \phi=3 a^{6}+9 a^{4} b^{2}+9 a^{2} b^{4}-9 a^{5}+9 \sqrt{3} a^{4} b-18 a^{3} b^{2}+10 \sqrt{3} a^{2} b^{3}-9 a b^{4} \\
& +12 a^{4}-18 \sqrt{3} a^{3} b+18 a^{2} b^{2}-10 \sqrt{3} a b^{3}-9 a^{3}-9 a b^{2}+9 \sqrt{3} a^{2} b+3 a^{2} \\
& +3 b^{6}+\sqrt{3} b^{5}-2 b^{4}+\sqrt{3} b^{3}+3 b^{2} \text {. }
\end{aligned}
$$

This point $\eta$, by construction, belongs to the two circumferences $A_{3} C_{2} A_{1} C_{3}, A_{3} B_{2} A_{2} B_{3}$. It only remains to prove that $\eta$ belongs to the circumference $C_{3} B_{1} C_{1} B_{3}$. But when we compute the distance between $\eta$ and $O_{B C}$ we obtain exactly the same expression obtained above for $r_{B C}$.

This completes the proof.

\section{Acknowledgement}

I would like to thank Agustí Reventós Tarrida, Departament de Matemàtiques, Universitat Autònoma de Barcelona for his constructive criticism of this paper.

\section{References}

[1] Cerin, Z.: On Properties of Rectangular Hyperbolas. Geom. Dedicata 84 (2001), 41-47.

[2] Hatzipolakis, A.P., Yiu, P.: The Lucas Circles of a Triangle. Amer. Math. Monthly 108 (2001), 444-446.

[3] Kimberling, C.: Triangle Centers and Central Triangles. Congressus Nunerantium 129 (1998).

[4] Kimberling, C.: Encyclopedia of Triangle Centers. http://cedar.evansville.edu/ ck6/encyclopedia/.

[5] van Lamoen, F.: Morley Related Triangles on the Nine-Point Circle. Amer. Math. Monthly 107 (2000), 941-945.

[6] van Lamoen, F.: Triangle Centers Associated with Rhombi. Elem. Math. 55 (2000), 102-109.

[7] van Lamoen, F.: Euler Lines Concurrent on the Nine-Point Circle. Problem 10796 [2000,367]. Amer. Math. Monthly 108 (2001), 569.

[8] Oldknow, A.: The Euler-Gergonne-Soddy Triangle of a Triangle. Amer. Math. Monthly 103 (1996), 319-329.

[9] Vandeghen, A.: Soddy's Circles and the de Longchamps Point of a Triangle. Amer. Math. Monthly 71 (1964), 176-179.

[10] Wetzel, J.E.: Converses of Napoleon's Theorem. Amer. Math. Monthly 99 (1992), 339-351.

Blas Herrera Gómez

Departament d'Enginyeria Informàtica i Matemàtiques

Universitat Rovira i Virgili

43007 Tarragona, Spain

e-mail: bherrera@etse.urv.es 